\title{
¿Cobertura universal? Las barreras en el acceso a la salud para la población refugiada nicaragüense en Costa Rica
}

\author{
Universal coverage? Barriers to access to health care for the \\ Nicaraguan refugee population in Costa Rica.
}

\author{
Alberto Cortés Ramos \\ Universidad de Costa Rica \\ San José, Costa Rica \\ alberto.cortes@ucr.ac.cr \\ Adriana Fernández Calderón \\ Universidad de Costa Rica \\ San José, Costa Rica \\ adriana.fernandez_c@ucr.ac.cr
}

\begin{abstract}
RESUMEN. El presente artículo tiene como objetivo principal analizar las principales barreras de acceso a los servicios públicos de salud de la población refugiada nicaragüense que empezó a llegar a Costa Rica a partir de la crisis política de abril de 2018 en Nicaragua. Para ello, el estudio se desarrolla en tres partes: primero, se estudian las características del sistema de salud costarricense; segundo, se analizan la trayectoria y características de la inmigración nicaragüense en Costa Rica y en un tercer momento, se analiza el acceso a los servicios de salud para esta población a partir de dos fuentes: las nuevas directrices y circulares que se han delineado en respuesta a los nuevos flujos migratorios hasta inicios del 2020 y grupos focales con la población migrante y dos organizaciones que les brindan asistencia en el país.
\end{abstract}

PALABRAS CLAVE. Migración nicaragüense, Población Refugiada, Derechos Humanos, Acceso a Salud, Seguridad Social.

Este es un artículo de acceso abierto publicado bajo los términos de la Licencia Creative Commons 4.0 Internacional Atribución-NoComercial-SinDerivadas. 


\begin{abstract}
The main objective of this article is to analyze the main barriers to access public health services for the Nicaraguan refugee population that arrived in Costa Rica after the political crisis of April 2018 in Nicaragua. For this, the study is developed in three parts: first, the characteristics of the Costa Rican health system are studied; Second, the trajectory and characteristics of Nicaraguan immigration in Costa Rica are analyzed and, in a third moment, access to health services for this population is studied based on both, the new guidelines and circulars that have been outlined in response to the new migratory flows until the beginning of 2020, and focus groups with migrant population and two NGOs that provide them with assistance in the country.
\end{abstract}

KEYWORDS. Nicaragua migration, Refugee Population, Human Rights, Access to Health, Social Security.

\title{
INTRODUCCIÓN
}

Las movilizaciones humanas, de Nicaragua a Costa Rica, tienen una larga trayectoria histórica que se ha reflejado en una importante variedad de dinámicas migratorias, incluyendo migraciones estacionales, permanentes y semipermanentes y migraciones de refugio. Las primeras se han caracterizado por responder a estrategias de supervivencia económica y a procesos de inserción laboral, mientras que la última, responde a desplazamientos por conflictos político-militares, represión, persecución y violencia.

La intensidad de estos vínculos migratorios ha generado la producción de un espacio social transnacional tejido y alimentado por estas dinámicas migratorias, creando una interdependencia económica, social y cultural entre ambos países, interdependencia no ajena de contradicciones y conflictos. Por ejemplo, algunos elementos que caracterizan este espacio son la existencia de miles de hogares binacionales, o la dependencia de importantes agro cadenas globales de productos (entre otros, piña, banano, naranja, café, tubérculos) de la fuerza de trabajo estacional nicaragüense que ingresa al país en los ciclos de cosecha. Diversas fuentes señalan que se trata de cerca de 80 a 120 mil trabajadores, que se insertan en estas actividades agrícolas y de construcción, en condiciones laborales muy precarias, pues una parte importante de las empresas productoras no respetan la legislación laboral ni el gobierno ha supervisado de manera adecuada el cumplimiento de esta legislación en estas actividades (Carazo y Aravena, 2016; Voorend y Robles, 2016; Nuevo 


\section{Diario, 2017; Revista SUMMA, 2017).}

La segunda dinámica es la de la migración semipermanente y permanente, que incluye población que se inserta en actividades de servicios diversos, tales como seguridad privada, construcción $\mathrm{y}$, en el caso de las mujeres, actividades de cuido y servicio doméstico. Esta es la migración que captura de mejor manera el Censo (Sandoval y Bonilla, 2011) y la ENAHO (2017). Esta última, en 2017 encontró durante el primer trimestre a 291,258 nicaragüenses en Costa Rica. De esa cifra 194,223 tenían empleo y de estos el 17.1 por ciento se empleaba en el servicio doméstico (La Prensa, 2017).

Una tercera dinámica migratoria nicaragüense en Costa Rica es la que se inició a partir del estallido de la crisis política en Nicaragua en abril de 2018, y que generó un importante desplazamiento forzado de nicaragüenses a Costa Rica que huían de la persecución, represión y las violaciones sistemáticas a los derechos humanos, con lo que el volumen de personas que cruza la frontera aumentó a partir de ese año.

En términos de las características de la población, esta migración de refugio difiere del perfil de la migración laboral o económica. Al ser provocada por causas políticas, una parte importante de este flujo es de personas activistas ciudadanas, políticas, periodistas y defensoras de derechos humanos y estudiantes universitarias/os. Se trata de una población en edad de trabajar (PET), con un nivel de calificación educativa mayor que la de la migración laboral previa y también, por su experiencia de activismo, tienen más capacidad de voz (en el sentido de Hirschman (1969), capacidad de hacerse oír y de reclamar derechos), por lo tanto, conocen y reclaman sus derechos y buscan, a través de diversos mecanismos, tener acceso a servicios en el país.

Entre junio de 2018 y marzo de 2020 solicitaron refugio aproximadamente 2.900 personas mensualmente, para un total de cerca de 80 mil solicitudes de refugio en dos años, con la particularidad de que, según las estimaciones, alrededor de un tercio de estas solicitudes eran de migrantes que ya residían en Costa Rica en condición irregular y que buscan aprovechar a la figura de refugio para intentar obtener su documentación (Rivas y Regidor, 2020).

Este contexto ha supuesto enormes retos para Costa Rica. Por una parte, la política receptora de personas migrantes y de respeto a los derechos humanos que le ha sido reconocida históricamen- 
te, supone un compromiso por dar respuesta oportuna no solo a la necesidad de protección humanitaria que requiere la población, sino también de facilitar las condiciones necesarias para promover su integración local. Por otra parte, el incremento en el número de solicitudes ha evidenciado significativas restricciones por parte de la institucionalidad pública para atender la demanda de documentación en los plazos esperados y para propiciar el acceso a servicios para la población, lo que se constituye como una de las barreras más grandes para integrarse al país y poder ejercer una ciudadanía social plena ${ }^{1}$ (Añón, 2002).

Esta problemática ha sido especialmente evidenciada en los intentos de la población por acceder a servicios de salud, en donde se han enfrentado a la lentitud de la burocracia estatal, el exceso de trámites, el desconocimiento de los funcionarios sobre su condición de solicitantes y los mecanismos que les protegen y a la discriminación por parte de estos, en lo que la población llama "xenofobia institucionalizada" (Grupo focal 3 con población solicitante de refugio, comunicación personal, 11 de diciembre de 2019).

Las barreras de la institucionalidad pública que limitan el acceso servicios de salud para la población nicaragüense se enmarcan en un contexto de transformaciones y tensiones en el régimen de protección social del país, erosión del universalismo, contención del gasto público, endurecimiento de las políticas para la atención en salud para la población migrante y deterioro en la calidad de la calidad de servicios sanitarios (Voorend, 2019).

En este contexto, a pesar de Costa Rica cuenta con uno de los más amplios regímenes de política social entre los países en vías de desarrollo (Martínez Franzoni y Sánchez-Ancochea, 2013 en Voorend, 2019, p.10) y posee un sistema de salud que es considerado como el más universal de la región, la reciente movilización de población solicitante de refugio al territorio costarricense ha abierto una vez más el debate sobre las dinámicas de exclusión social que

1 Tal y como se discute en este artículo, asumimos que la ciudadanía social “... consiste en asegurar que cada cual sea tratado como un integrante pleno de una sociedad de iguales (Marshall, 1960). La ciudadanía es entendida como status conformado por el acceso a los recursos básicos para el ejercicio de derechos y deberes. La no discriminación en el acceso a esos recursos constituye la condición necesaria y suficiente de la ciudadanía. De forma que la ciudadanía en su sentido más pleno precisa un modelo de estado del bienestar democrático." (Marshal (1960) en: Añón, 2002) 
el Estado costarricense propicia. Esto no solo por la incapacidad de dar respuesta a las solicitudes para acceder a documentación en los plazos establecidos, sino también a través de la obstrucción y las barreras que impiden que la población pueda navegar por la institucionalidad pública y acceder a servicios de salud.

A partir de estos elementos, el presente artículo se estructura en tres partes: primero, analiza las características del sistema de salud costarricense; segundo, caracteriza los rasgos de la inmigración nicaragüense a Costa Rica y en un tercer momento, analiza el acceso a los servicios de salud para esta población a partir de las nuevas directrices y circulares que se han delineado en respuesta a los nuevos flujos migratorios hasta inicios del 2020.

\section{METODOLOGÍA}

Con el fin de recopilar las percepciones de la población migrante sobre el proceso para acceder a servicios de salud y mapear las barreras que encuentran durante la ruta, para esta investigación se aplicaron grupos focales y técnicas de investigación-acción participativa de mapeo participativo y cartografía corporal.

Se realizaron un total de cuatro grupos focales. Dos de ellos, con población solicitante de refugio masculina y femenina, de nacionalidades nicaragüense, venezolana y hondureña, con edades entre los 20 y los 60 años, la mayoría con secundaria completa y en algunos casos con estudios universitarios avanzados, que se encuentra viviendo en la Gran Área Metropolitana. La mayoría de estas personas alquilan propiedades como medio de vivienda. El objetivo fue conocer cuál ha sido la experiencia de la población en el proceso de acceder a servicios, cómo han resuelto los obstáculos durante la ruta y cuáles instituciones u organizaciones no gubernamentales les han brindado orientación y apoyo durante el proceso. Asimismo, se realizaron otros dos grupos focales con representantes de las organizaciones RET Internacional y el Centro de Derechos Sociales del Inmigrante (Cenderos), que trabajan directamente con esta población para reconstruir, más allá de la normativa institucional, cómo operan las rutas de acceso a salud en las vivencias diarias de la población. Estos cuatro grupos focales fueron ejecutados en el marco del proyecto de acción social de la Universidad de Costa Rica "Acompañar para integrar: rutas para promover la integración local de la población migrante en Costa Rica.

\section{1}


Aparte, se aplicaron técnicas de mapeo participativo y cartografía corporal en el marco de los cursos de formación sobre "Ciudadanía, derechos humanos, migración y refugio de la Universidad de Costa Rica", a un total de 33 personas, de las cuales 14 eran mujeres y 19 eran hombres, solicitantes de refugio, mayores de edad, especialmente estudiantes, activistas sociales o profesionales. El mapeo participativo tuvo como propósito, conocer desde la perspectiva y experiencia del grupo, cuáles eran los principales problemas que habían enfrentado en su inserción, destacando la regularización, el empleo y el acceso a la salud, como los principales problemas señalados y en ese orden. La cartografía corporal permitió aproximarse a la vivencia más personal, incluso corporal, del impacto de los problemas enfrentados por el grupo tanto en su salida de Nicaragua como en su sobrevivencia en Costa Rica, destacando el stress, la angustia, la tristeza, pero también la esperanza, como elementos que fueron indicados por los subgrupos en los materiales que trabajaron.

Finalmente, se recurrió al análisis documental de las directrices y circulares que regulan el acceso a la salud de la población refugiada y solicitante a partir de abril de 2018, esta documentación fue suministradas por las organizaciones que trabajan con la población y constituye los instrumentos normativos que utilizan para orientarles en el proceso de acceder a la salud

\section{SISTEMA DE SALUD EN COSTA RICA: UNA TRAYECTORIA UNIVERSALISTA Y SOLIDARIA CON MATICES}

El sistema de salud costarricense se ha caracterizado históricamente por tener una trayectoria universalista y solidaria, que se refleja en la cobertura masiva y la amplia gama de servicios que llegan a prácticamente a todos los estratos de la población. Este modelo le ha permitido al país alcanzar resultados en salud que son solo comparables con países de un ingreso mayor y consolidarse como uno de los sistemas más universales de la región latinoamericana (Alvarenga et al., 2018).

Los resultados favorables en materia de salud fueron posibles gracias a la creación de la Caja Costarricense de Seguro Social (CCSS) en 1941 y la apuesta por impulsar un seguro social obligatorio y financiado de forma tripartita con los aportes de los emplea- 
dos, los empleadores y el Estado (Miranda, 1994). La expansión se hizo inicialmente de manera ascendente, con el aseguramiento de los grupos urbanos con ingresos menores y posteriormente incorporando a los de más altos ingresos (Miranda 1994).

La lógica universal del sistema de salud se fortaleció durante la década de 1970, con el impulso de varias reformas, entre ellas, la ruptura de los límites máximos de la cotización - con lo que se incorporó a los sectores con mayores ingresos al sistema de cotización, la ampliación de manera gradual de la cobertura del seguro social para los trabajadores independientes y la inclusión en los servicios de salud de las personas en situación de pobreza por medio de programas de salud rural y comunitaria financiados por el Fondo de Desarrollo y Asignaciones Familiares (FODESAF) creado en 1975. Las decisiones para incorporar estos nuevos sectores al sistema de salud permitieron a Costa Rica convertirse en un caso excepcional de universalización en el sur global (Martínez Franzoni y Sánchez-Ancochea, 2016).

Asimismo, durante esta década se aprobó una reforma constitucional que acordó universalizar los servicios (Rosemberg, 1979) y se decidió traspasar a la CCSS a los hospitales que estaban a cargo de otras instituciones, con lo que se alcanzó una mayor unificación de la oferta de servicios (Martínez Franzoni y Sánchez-Ancochea, 2016). A pesar de todos estos avances que robustecieron el sistema de salud y su cobertura universal, entre 1980 y la década de 1990, las decisiones impulsadas para dar respuesta a la crisis económica tuvieron importantes repercusiones sobre el modelo de seguridad social, interrumpieron la tendencia expansionista (Martínez Franzoni y Sánchez-Ancochea, 2016) y cimentaron cambios que amenazarían la trayectoria universalista.

Para entender estas transformaciones en el sistema de salud, es necesario hacer referencia al concepto de universalismo desarrollado por Martínez Franzoni y Sánchez-Ancochea (2016), que lo definen en términos de los resultados deseables de la política pública. Estos autores proponen tres dimensiones: cobertura, eficiencia y equidad. Se dice que las políticas son universales cuando tienen cobertura masiva, es decir que los beneficios alcanzan a la mayoría de la población en una categoría; la suficiencia refiere al nivel y la calidad de los servicios: cuanto más exhaustivos sean y de mayor calidad, más universal es la política; y finalmente la equidad refiere a la distribución igualitaria de los beneficios.

\section{3}


En términos generales, la crisis económica desencadenó una reducción del gasto en salud y disminuyó la inversión en infraestructura y el equipamiento, a partir de una disposición legal aprobada que impedía los aumentos de inversión en la CCSS (Martínez Franzoni y Sánchez-Ancochea, 2016). Asimismo, se impulsó una reforma al sector salud, con el objetivo de incrementar la eficiencia en los hospitales y los centros de salud que, si bien amplió la gama de servicios, recortó los suministros básicos generando un aumento en las listas de espera y un debilitamiento en la calidad de la atención (Martínez Franzoni y Sánchez-Ancochea, 2013 en Voorend, 2019).

Durante el 2011, la CCSS enfrentó una crisis financiera sin precedentes como resultado de malas decisiones gerenciales, los efectos económicos de la crisis económica del 2009 sobre el gasto en salud, el aumento de los costos de las nóminas y corrupción (Carrillo et al., 2011; Jaramillo, 2013; Mora, 2015; OPS, 2011 en Voorend, 2019), por lo que su sostenibilidad financiera se encuentra hoy amenazada.

A pesar de que en el 2014 la cobertura en los servicios de salud alcanzó el 95\% de la población (OCDE, 2018), la suficiencia y la equidad en los servicios han sufrido deterioro. Lo anterior se refleja en la insatisfacción de los usuarios con la calidad de los servicios y en el aumento sustantivo en la búsqueda de opciones privadas. De acuerdo con los datos de la Organización para la Cooperación y el Desarrollo Económicos (OCDE), Costa Rica ha reportado un crecimiento de un $18,71 \%$ a un $24,87 \%$ en el gasto de bolsillo como porcentaje del gasto total en salud ${ }^{2}$ y la encuesta de la Contraloría General de la República (Encuesta Nacional de Percepción de los Servicios Públicos, 2018), señala que un $76.5 \%$ de los costarricenses encuestados reportaron haber incurrido en gastos adicionales para acceder a servicios privados de salud (Contraloría General de la República, 2018).

De acuerdo con la OCDE, la tendencia del gasto directo de bolsillo sugiere que en Costa Rica se está desarrollando un sistema dual, en el que un número significativo de personas está acudiendo a los servicios privados para consultas médicas. De acuerdo con la organización, la existencia de un sistema dual sería un fallo mayor para la tradición solidaria y de servicios básicos pagados con fon-

$2 \mathrm{El}$ gasto de bolsillo se refiere al peso relativo de los pagos directos que hacen los hogares en salud respecto al gasto total del país en este rubro. 
dos públicos que ha mantenido Costa Rica (OCDE, 2018).

Uno de los factores que motiva a la población a hacer uso de la opción privada, son las largas listas de espera en el sistema público. De acuerdo con los datos disponibles para el 2019, los pacientes en la Caja tienen que aguardar un promedio de 369 días para una cirugía, 171 días para un procedimiento médicos y 200 días para recibir una consulta externa (Cordero Parra, 2019), sin embargo, estos datos son estimaciones, ya que hasta ahora estas estadísticas no son públicas $^{3}$. Con el fin de dimensionar la magnitud del problema a continuación se comparten algunos datos relativos a las listas de espera quirúrgicas electivas (aquellas que requieren hospitalización) en varios hospitales públicos. Estos datos fueron solicitados por parte del Poder Judicial a la CCSS.

Tabla 1. Número total de personas y plazo de espera promedio en listas quirúrgicas electivas de las especialidades de ortopedia, cirugía general y urología en tres hospitales públicos de Costa Rica a setiembre de 2018

\begin{tabular}{llll}
\hline Especialidad & Hospital & $\begin{array}{l}\text { Cantidad de } \\
\text { pacientes }\end{array}$ & $\begin{array}{l}\text { Plazo promedio } \\
\text { de espera en días }\end{array}$ \\
Ortopedia & $\begin{array}{l}\text { Hospital Rafael } \\
\text { Ángel Calderón } \\
\text { Guardia }\end{array}$ & 1682 & 371 \\
Cirugía general & $\begin{array}{l}\text { Hospital Rafael } \\
\text { Ángel Calderón } \\
\text { Guardia }\end{array}$ & 1078 & 609 \\
Urología & $\begin{array}{l}\text { Hospital Rafael } \\
\text { Ángel Calderón } \\
\text { Guardia }\end{array}$ & 928 & 542 \\
Ortopedia & $\begin{array}{l}\text { Hospital San Juan } \\
\text { de Dios }\end{array}$ & 2564 & 717 \\
Cirugía general & $\begin{array}{l}\text { Hospital San Juan } \\
\text { de Dios }\end{array}$ & 1559 & 312 \\
Urología & $\begin{array}{l}\text { Hospital San Juan } \\
\text { de Dios }\end{array}$ & 686 & 331 \\
\hline
\end{tabular}

3 En el 2019 se presentó un decreto para hacer públicas las listas de espera. De acuerdo con el ministro de Salud, Daniel Salas, se requiere transparencia y acceso a la información por parte de la población para conocer cuantificaciones de las listas de espera por servicios, por procedimientos e internamientos en los diferentes hospitales (Hidalgo, 2019). 


\begin{tabular}{llll} 
Ortopedia & Hospital México & 1364 & 865 \\
Cirugía general & Hospital México & 2389 & 591 \\
Urología & Hospital México & 217 & 204 \\
\hline
\end{tabular}

Fuente: Elaboración propia con base en datos de la resolución № 05560 - 2019 de la Sala Constitucional (Resolución № 05560 - 2019 2019)

La insatisfacción de la población con los servicios de salud ha quedado constatada en algunas encuestas y en la cantidad de recursos de amparo que han interpuesto ante la Sala Constitucional para demandar una atención oportuna. De acuerdo con la Encuesta Nacional de Percepción de los Servicios Públicos, aproximadamente una de cada tres personas considera que no se brindan suficientes citas por día en los Equipos Básicos de Atención Integral en Salud (EBAIS) $(68 \%)$, dos de cada cinco entrevistadas reportan que son insuficientes en las clínicas (59\%) y más de la mitad en los hospitales (53\%). Asimismo, el $47 \%$ de la población reporta que tuvo que esperar más de un año para ser atendido en el centro de salud (Contraloría General de la República 2018). Por otra parte, mientras que en el año 2012 se presentaron 1.745 recursos de amparo por violaciones al derecho a la salud, esta cifra ascendió a 7.037 recursos en el 2018 (La Nación, 2018).

Gráfico 1. Recursos de amparo por violaciones al derecho a la salud en el periodo 2012-2018

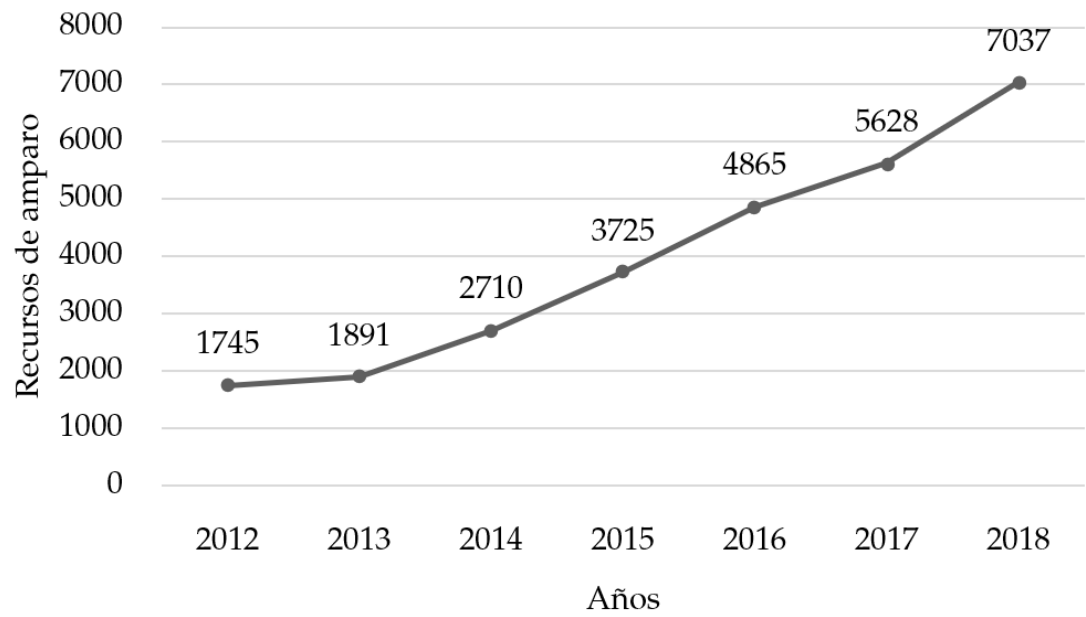


Fuente: Elaboración propia con base en datos de Recio (2019).

En este contexto, la aspiración universal y solidaria del sistema, se encuentra en entredicho por el aumento de personas recurriendo a la opción privada para tener acceso a servicios de salud, sino también, cómo se verá más adelante por las restricciones que enfrenta un sector de la población, en este caso la solicitante y refugiada para acceder a los servicios y las transformaciones que ha sufrido el seguro social en cuanto a su orientación de servicio que afectan tanto a costarricenses como a extranjeros.

De acuerdo con los datos de la OCDE (2017) aproximadamente el $5 \%$ de la población continúa sin estar registrada en el seguro social, este grupo incluye a trabajadores informales, especialmente de países vecinos, a población refugiada a los que no se le brindan servicios por cargos al Estado (algunos tienen seguros médicos cubiertos por el Alto Comisionado de las Naciones Unidas para los Refugiados, ACNUR), inmigrantes indocumentados y algunos grupos indígenas.

De esta forma, la población nicaragüense, tanto la migrante económica como la solicitante de refugio en Costa Rica, intenta acceder a servicios de salud en un contexto de significativas transformaciones de la seguridad social que se han visto traducidas en restricciones presupuestarias, desmejoramiento en la calidad de los servicios, deterioro del proyecto universalista, así como una "xenofobia institucionalizada", como lo llama la población (Grupo focal 3 con población solicitante de refugio, comunicación personal, 11 de diciembre de 2019).

\section{CARACTERIZACIÓN BÁSICA DE LAS DINÁMICAS MIGRATORIAS DE NICARAGUA HACIA COSTA RICA}

Desde la perspectiva de la larga duración, un elemento distintivo y continuo de la relación migratoria entre estos dos países es que la dirección de los flujos ha sido de norte a sur. Hay dos factores claves que contribuyen a explicar esa orientación: primero, la temprana y duradera estabilidad política de Costa Rica, incluyendo la consolidación de un régimen democrático a lo largo del siglo XX, y frente a la inestabilidad geopolítica, la prevalencia de regímenes autoritarios y la legitimidad de la violencia como mecanismo para 
resolver diferencias en Nicaragua. Segundo, una dinámica de complementariedad entre los mercados laborales de ambos países, con varios períodos en los que la economía costarricense ha requerido atraer fuerza de trabajo extranjera, frente a situaciones en las que Nicaragua más bien estaba inmersa en procesos que generaban expulsión de su población (Cortés Ramos, 2009; Morales Gamboa, 2007).

De tal forma que, desde finales del siglo XIX, los ciclos migratorios de Nicaragua hacia Costa Rica se han alternado, combinando migraciones de carácter político con las de carácter económico o laboral (estacional y permanentes). Una rápida periodización de estos ciclos iniciaría con la migración laboral para trabajar en la construcción del ferrocarril primero y en el enclave bananero después, a finales del siglo XIX y hasta mediados del siglo XX (Cortés Ramos, 2009). A lo largo de la dinastía somocista (1936-1979) hubo recepción de asilados políticos en Costa Rica, pero esta migración no adquirió importancia sino hasta la década del setenta del siglo pasado, cuando la represión de la dictadura aumentó y el FSLN utilizó a Costa Rica como retaguardia estratégica. La información existente indica que al momento del triunfo de la Revolución en 1979 habían cerca de 80 mil migrantes nicaragüenses y una década después, habían más de 120 mil nicaragüenses, de los cuales cerca de 40 mil tenían condición de refugiados. Una mayoría de esta población regresó a Nicaragua en 1990, después del triunfo de doña Violeta, con la expectativa de vivir en democracia y en paz (Morales Gamboa, 2007; Cortés Ramos, 2009).

Las transformaciones producidas en Nicaragua a inicios de los 1990s por el cambio de régimen político y económico (i.e.: privatizaciones de instituciones públicas, desarme de las fuerzas contrarrevolucionarias y la reducción del ejército nicaragüense), pronto generó un nuevo ciclo de expulsión de población que se acopló con las transformaciones económicas que estaban sucediendo en Costa Rica y que implicaba un aumento de la demanda laboral en el sector agrícola de exportación, construcción, seguridad privada y empleo doméstico. Este importante flujo de migración laboral era rural y urbana y ha sido la más masiva de todos los ciclos migratorios (Cortés Ramos, 2009; Morales Gamboa, 2007; Voorend y Robles, 2016).

Las dinámicas de migración laboral se mantuvieron en las últimas dos décadas, aunque el tamaño se fue reduciendo con el paso 
del tiempo. Según el último censo (2011), la población nicaragüense en Costa Rica alcanzaba las 287.766 personas. Según ENAHO (2019), la población nicaragüense permanente ya había alcanzado las 376.611 personas. Como se sabe, los censos no incluyen a la población que tiene menos de seis meses de estar en el país y por ello no registra a la migración estacional que trabaja en el área rural por períodos cortos y que, como se indicó al inicio del artículo, se calcula que oscila entre $80 \mathrm{mil}$ y $120 \mathrm{mil}$ personas. El censo también tiene dificultades para medir la migración irregular (Proyecto Estado de la Nación, 1999). De manera tal que, es posible proyectar que la migración nicaragüiense en Costa Rica, tomando en cuenta las distintas dinámicas migratorias, puede estar cerca del medio millón de personas, o sea, aproximadamente un 10 por ciento del total de la población del país (Brenes et al, 2000)․

\section{CRISIS POLÍTICA DE ABRIL DE 2018 Y MIGRACIÓN DE REFUGIO DE NICARAGUA HACIA COSTA RICA}

La crisis política de abril de $2018^{5}$ en Nicaragua inició un nuevo ciclo de migración hacia Costa Rica. Esta crisis se desarrolló a partir de un decreto con reformas a la seguridad social publicado por el gobierno de Nicaragua, que gravaba las pensiones (Chamorro Barrios, 2020). El 19 de abril, grupos de estudiantes universitarios, activistas de sociedad civil, feministas y jubilados de la tercera edad convocaron en Managua y León a protestas cívicas que fueron reprimidas violentamente por fuerzas de choque del FSLN acompañadas por la Policía Nacional, la cual les dejó actuar con impunidad. Esta represión fue transmitida en vivo por más de tres horas en televisión y se viralizó en redes sociales. Ello dio pie a una incorporación masiva de estudiantes universitarios en la protesta en los días siguientes, que incluyó la toma de las universidades públicas. La reacción del régimen no se hizo esperar, aumentando el accionar violento de los grupos paramilitares y de la Policía Nacional. Conforme fue aumentando el accionar represivo, los asesinatos, los encarcelamientos $y$, en general, la violencia del régimen de Orte-

4 En este artículo, Brenes et. al. Desarrollan una metodología para calcular el número de inmigrantes nicaragüenses.

5 Hay un completo recuento de lo sucedido a partir del 18 abril de 2018 y sus antecedentes en el libro Anhelos de un nuevo horizonte: Apuntes para una Nicaragua democrática, compilado por Cortés Ramos, et al (2020). 
ga-Murillo contra la oposición cívica, la salida de activistas a Costa Rica empezó a darse, de manera clara a partir de junio de 2018.

Para dar una idea del cambio en las solicitudes de refugio, entre enero y marzo de 2018 hubo 22. En abril hubo 24 y en mayo, 87. A partir de junio, después de los asesinatos con francotiradores de la marcha de las madres ${ }^{6}$ el 30 de mayo y la represión violenta de los tranques en las carreteras, la cifra de solicitudes pasó a 3.344 en junio (Valverde, 2018 y a 5.279 en julio de ese año. Entre junio de 2018 y marzo de 2020 solicitaron refugio un promedio mensual de 2.900 personas, para un total de cerca de 80 mil solicitudes de refugio en dos años. ${ }^{7}$

\section{RUTAS DE ACCESO A LOS SERVICIOS DE SALUD PARA LA POBLACIÓN MIGRANTE, REFUGIADA Y SOLICITANTE}

Actualmente, la CCSS es la institución pública encargada de la administración, prestación y gestión de los servicios de salud y tiene a su cargo los tres niveles de atención: el primario organizado en torno a los EBAIS, el secundario conformado por clínicas periféricas y hospitales regionales y el terciario integrado por hospitales nacionales (Mohs, 1983). En el primer nivel de atención, la población recibe servicios acudiendo a los EBAIS según su domicilio. Hay un total de 1.014 EBAIS (Solís Ramírez, 2020), extendidos en todo el territorio nacional (Solís Ramírez, 2020), y funcionan en red con los centros definidos en el segundo nivel de atención, es decir las personas son referidas desde la atención primaria a la secundaria (OCDE, 2017). Para recibir atención en los EBAIS, se requiere contar con alguno de los distintos tipos de seguro que administra la Caja (Voorend, 2019). Las modalidades de aseguramiento en el país son tanto contributivas, como no contributivas. En el grupo de las primeras se encuentra el aseguramiento obligatorio para trabajadores asalariados, en el que contribuyen con aportes el trabajador,

6 Se trató de una manifestación encabezada por las madres de las víctimas de la represión de abril en Nicaragua. Durante la marcha, grupos parapoliciales y oficiales antidisturbios dispararon contra los manifestantes. La represión dejó quince personas muertas y 79 heridas.

7 Dirección General de Migración y Extranjería. (2020). Informes de Unidad de Refugio mayo 2018-abril 2020. San José: Unidad de Refugio, Dirección General de Migración y Extranjería. 
el contratante y el Estado (Voorend, 2019). Asimismo, existe el aseguramiento para trabajadores independientes que cotizan de forma individual, el aseguramiento voluntario para quienes carecen de un trabajo remunerado y el aseguramiento familiar que cubre a los familiares de los asegurados directos asalariados, pensionados o por cuenta propia (Picado y Salazar, 2004).

En el segundo grupo se encuentra el aseguramiento por cuenta del Estado, que incluye a la población de escasos recursos económicos y que no tiene la posibilidad de asegurarse bajo alguna de las otras modalidades (Picado y Salazar, 2004). Como parte de esta modalidad, se cubre a la población a la que la Caja le presta servicios por estar cubierta por leyes y normas específicas establecidas por el Estado, como es el caso de los niños de 0 a 6 años y las personas que se encuentran en edad escolar inscritas en el sistema educativo hasta los 18 años (Voorend, 2019). Los únicos servicios cuyo acceso no depende de una modalidad de aseguramiento, son los de urgencias, sin embargo, están sujetos a cobro posterior a la prestación de la atención (Picado y Salazar, 2004). De esta forma, cuando una persona migrante obtiene cualquier modalidad de aseguramiento de las anteriormente mencionadas, tiene acceso a los mismos servicios que los nacionales y a la cobertura completa que ofrece el sistema.

Sin embargo, a partir de la entrada en vigor de la Ley General de Migración y Extranjería en 2010 y su reglamento, y la consecuente aprobación de una serie de políticas a lo interno de la Caja, el acceso a la salud se ha obstaculizado. Estaa nueva legislación establece que "la tramitación de toda gestión migratoria deberá garantizar el aseguramiento a la seguridad social por parte de las personas migrantes. Tal garantía obligará a que todo trámite migratorio deba contemplar, como uno de sus requisitos básicos, contar con los seguros que brinda la Caja Costarricense de Seguro Social (CCSS)" (artículo 7, inciso 7). De forma paralela, la Caja estableció en el 2012, una disposición que indica que las personas extranjeras que soliciten aseguramiento para poder renovar su documento migratorio deben presentar la cédula de residencia vigente o bien demostrar que el proceso para regularizarse se encuentra en estado de aceptación y siendo procesado, lo que faculta a la CCSS a emitir un seguro médico temporal (Voorend, 2019). De acuerdo con Voorend (2019), estas decisiones se establecen como una trampa que dificulta el proceso de regularización al exigir seguros para renovar 
la documentación, al mismo tiempo que se demande la regularización del estado migratorio para acceder al seguro.

Según Voorend (2019), la Sala Constitucional decretó que la Dirección General de Migración y Extranjería (DGME) debe conceder un documento temporal que facilite a la población migrante para iniciar su proceso de aseguramiento. Sin embargo, continúa Voorend (2019), en la práctica se muestra que esta disposición no resolvió la dificultad, ya que, en algunos casos, en el sitio web no se especifica que la población tiene la posibilidad de optar por esta alternativa. Asimismo, existen casos en los que la población de forma previa ha tenido que recurrir a los servicios de salud $\mathrm{y}$, al no contar para ese momento con aseguramiento, terminen con facturas pendientes. Así, aunque intenten asegurarse no pueden hacerlo y esto a su vez repercute con que puedan renovar su documento. Se trata entonces de medidas más severas impuestas por la institucionalidad pública que impiden el acceso a los servicios, ya que en el pasado las personas migrantes podían asegurarse con menores requisitos (Voorend, 2019).

A partir de 2018, con lo cambios en los flujos migratorios, además de las distintas modalidades de aseguramiento que se configuran como criterios de elegibilidad para hacer uso de los servicios de salud, la atención está regulada por una serie de directrices, circulares y oficios, que cambian de manera dinámica el tiempo, y en las que, según reportan las organizaciones que atienden esta población, se erigen oportunidades para acceder a los servicios, que en la práctica, no siempre se ven consagradas debido a dinámicas institucionales que imponen barreras de acceso (grupo focal 1 con población solicitante de refugio, grupo focal 2 con representantes de organización que trabaja con migrantes y grupo focal 3 con población solicitante de refugio, comunicación personal, 2 de octubre de 2019, 7 de octubre de 2019 y 11 de diciembre de 2019).

Desde junio de 2018, se encuentra vigente la directriz 010-MPMIDEPLAN-MTSS-MSP-MGP-MRREE, emitida por el Ministerio de la Presidencia en conjunto con otras carteras ministeriales, en la que se establecen los lineamientos para dar respuesta a los flujos migratorios que ingresen o permanezcan en nuestro país en situaciones especiales o excepcionales, tales como tránsito, condición irregular, refugio, tráfico ilícito de migrantes o trata de personas. 
Tabla 2. Directrices, oficios y circulares que establecen los derechos y lineamientos del acceso a servicios de salud de la población migrante y solicitante de refugio

\begin{tabular}{|c|c|}
\hline Normativa & Disposiciones generales \\
\hline $\begin{array}{l}\text { Directriz 010-MP- } \\
\text { MIDEPLAN- } \\
\text { MTSS-MSP-MGP- } \\
\text { MRREE }\end{array}$ & $\begin{array}{l}\text { Establece que la población migrante tiene derecho a } \\
\text { recibir atención básica en salud por enfermedades y } \\
\text { dolencias de acuerdo con el marco jurídico vigente y que } \\
\text { el costo está a cargo del Estado. }\end{array}$ \\
\hline $\begin{array}{l}\text { Anexo oficio GM- } \\
\text { D-10.791-2018- } \\
\text { / GF-4033-2018 }\end{array}$ & $\begin{array}{l}\text { Para facilitar el acceso a salud, los funcionarios deben } \\
\text { verificar que la persona cuente con un seguro. En el caso } \\
\text { de mujeres embarazadas y menores de edad se puede } \\
\text { generar una factura con cargo al Estado y para las demás } \\
\text { personas, si no cuentan con un seguro, se genera una } \\
\text { factura a su nombre. Asimismo, se establece que la aten- } \\
\text { ción integral de la población será financiada por el Fondo } \\
\text { Especial de Migración, el Fondo Social Migratorio y el } \\
\text { Fondo Nacional Contra la Trata de Personas y el Tráfico } \\
\text { Ilícito de Migrantes. }\end{array}$ \\
\hline ACE-157-08-18 & $\begin{array}{l}\text { Las personas menores de edad, mujeres embarazadas, } \\
\text { personas con tratamiento obligatorio y enfermedades } \\
\text { deben ser atendidos y la factura se hace con cargo al } \\
\text { Estado. Los usuarios identificados por REDES } \\
\text { grantes como mi- mayos de edad, sin capacidad de pago pasarán } \\
\text { a la Unidad de Validación y Servicios Médicos para la } \\
\text { facturación correspondiente } \text { f }^{9}\end{array}$ \\
\hline ACE-128-07-2019 & $\begin{array}{l}\text { Se reitera que la población migrante deberá recibir aten- } \\
\text { ción básica en salud y que para recibirlo se les identifica- } \\
\text { rá en los centros de salud con el pasaporte, documento o } \\
\text { carné entregado por la Dirección Nacional de Migración } \\
\text { Extranjería y que se factura de acuerdo con lo instruido } \\
\text { en el anexo GM-D-10.791-2018 y la circular ACE-157-08- } \\
18 \text {. }\end{array}$ \\
\hline
\end{tabular}

Fuente: Elaboración propia con base en la directriz 010-MP-MIDEPLAN-MTSS-MSP-MGPMRREE, el anexo oficio GM-D-10.791-2018 del Poder Ejecutivo y las circulares de la CCSS (ACE-157-08-18 y ACE-128-07-2019).

En dicha directriz, su anexo y las posteriores circulares emitidas por la CCSS (ACE-157-08-18 y ACE-128-07-2019) (véase tabla 2) para su efectivo cumplimiento, se reitera que los menores de edad

8 La sección de Registros Médicos de la Caja Costarricense del Seguro Social se encarga de identificar a cada usuario con el pasaporte, documento o carné otorgado por la Dirección General de Migración y Extranjería o el número interno que le asigne el establecimiento de salud.

9 La sección de Registros Médicos de la Caja Costarricense del Seguro Social se encarga de identificar a cada usuario con el pasaporte, documento o carné otorgado por la Dirección General de Migración y Extranjería o el número interno que le asigne el establecimiento de salud.

\section{3}


y las mujeres embarazadas, de conformidad con lo establecido en el Código de la Niñez y Adolescencia, tienen prioridad de atención y esta no puede ser negada. Asimismo, se establece que, a las personas con enfermedades de tratamiento obligatorio y enfermedades de vectores, se les brindará la atención y el cobro se hará bajo la modalidad de cargos al Estado. Finalmente, para las personas que no entran en estas categorías y no poseen capacidad de pago, cuando se trate de servicios del primer nivel de atención, se emitirá una factura a su nombre y se cubrirá bajo las disposiciones establecidas la directriz 010-MP-MIDEPLAN-MTSS-MSP-MGP-MRREE ${ }^{10}$.

De acuerdo con las organizaciones que trabajan con la población migrante (grupos focales 2 y 4 con organizaciones que asisten a migrantes, comunicación personal, 2 de octubre de 2019 y 3 de febrero de 2020), en la práctica, en algunos casos se harán los cobros y en otros no. Lo anterior tiene que ver con el conocimiento que tengan los funcionarios de las directrices que establecen el derecho a la atención en el primer nivel y el posterior proceso de emisión de factura a nombre de la persona migrante, pero que finalmente se financiará bajo lo dispuesto en la directriz 010-MP-MIDEPLANMTSS-MSP-MGP-MRREE. Asimismo, señalan que algunos funcionarios se amparan en el Reglamento del Seguro de la CCSS establece en el artículo 61 que "(1)os servicios que se otorguen a personas extranjeras no aseguradas en condición de pobreza a quienes no les puede ser otorgado el Seguro por el Estado, deberán facturarse para efectos del cobro a la instancia de Gobierno que corresponda". En casos como estos la factura se emite a la Dirección General de Migración y Extranjería, con cargo al Fondo Social Migratorio ${ }^{11}$, de manera que existe la posibilidad de que la atención sea financiada

10 En el artículo 5 de la directriz se establece que las acciones necesarias para la ejecución del "Plan de Atención Integral" (incluyendo la atención en salud) se financiarán, en primer término, por medio del Fondo Especial de Migración, el Fondo Social Migratorio y el Fondo Nacional contra la Trata de Personas y el Tráfico Ilícito de Migrantes, de acuerdo con la normativa específica y posibilidad presupuestaria de cada fondo.

11 El Fondo Social Migratorio fue creado para apoyar el proceso de integración de la población migrante y se financia a partir de un cobro adicional que se les hace durante los trámites de regularización migratoria, específicamente se trata de veinticinco dólares estadounidenses que se les cobra a las personas extranjeras en el momento en que se otorgue su regularización migratoria o cuando renuevan su permanencia en el país (Ley General de Migración y Extranjería, artículo 33, inciso 4). 
bajo esta modalidad. Por otra parte, y como se desarrollará más adelante en los hallazgos sobre los grupos focales realizados para esta investigación, esta decisión está permeada por un componente de discrecionalidad, ya que, en muchos casos, el cobro queda a voluntad del funcionario y de la jefatura de cobro y la posibilidad de la persona de probar su situación de pobreza, lo que explica por qué algunas personas reportan que recibieron el cobro por los servicios y otras no.

Uno de los aspectos más relevantes de las circulares que emite la CCSS en cumplimiento a la directriz mencionada anteriormente, es que la población puede acceder al primer nivel de atención sin tener ningún tipo de aseguramiento; pero es a partir del segundo y tercer nivel, en los que, ante ausencia de algunas de las modalidades de seguro, tienen que pagar la factura correspondiente de los servicios recibidos. Sin embargo, en la práctica, muchas veces los funcionarios desconocen estas disposiciones y niegan el acceso a los servicios en los EBAIS, lo que se traduce en que padecimientos que son tratables o que pudieron haber sido diagnosticados a tiempo, se conviertan luego en problemas más complejos y difíciles de tratar (grupo focal 4 con representantes de organización que trabaja con migrantes, comunicación personal, 3 de febrero de 2020).

De esta forma, según reportan las organizaciones que trabajan en la atención de la población refugiada, las circulares emitidas a partir de la directriz en principio muestran que existen varios mecanismos de acceso que muchas veces no se ejecutan. Asimismo, señalan que la ruta para acceder a los servicios de salud es sumamente engorrosa y que la provisión de servicios depende del conocimiento o no que tengan los funcionarios sobre las distintas directrices, pero también de si la población es o no debidamente orientada sobre los pasos que debe seguir para acceder a ellos (grupo focal 1 con población solicitante de refugio, grupo focal 2 con representantes de organización que trabaja con migrantes y grupo focal 4 con representantes de organización que trabaja con migrantes, comunicación personal, 2 de octubre de 2019, 7 de octubre de 2019 y 11 de diciembre de 2019 ).

En febrero de 2020, se firmó un convenio entre la CCSS y el ACNUR, para asegurar a aproximadamente 6.000 personas refugiadas y solicitantes en condiciones de extrema vulnerabilidad (Alto Comisionado de Naciones Unidas para los Refugiados, 2020). Si bien, este acuerdo ha facilitado la ruta de acceso a los servicios en los tres

\section{5}


niveles de atención, al estar orientado a un grupo específico, este artículo no se concentrará en su análisis, sino que en el de los casos de las personas que continúan tratando de acceder a partir de las regulaciones establecidas en las directrices y circulares.

En este sentido, resulta no solo relevante analizar cuáles son los lineamientos de la normativa, sino también reconstruir lo que ocurre con las rutas de acceso a partir de las vivencias de las personas mientras navegan por la institucionalidad pública. Desarrollar investigación en esta materia cobra especial relevancia en un contexto en el que se registran nuevas respuestas institucionales en atención a los flujos migratorios y, por lo tanto, se configuran nuevas rutas para acceder a los servicios. El aporte de esta investigación reside en dar cuenta de estas nuevas dinámicas en relación con el acceso a la salud, a partir de las directrices y circulares emitidas después de abril de 2018.

\section{NAVEGAR EN LA SEGURIDAD SOCIAL: PERCEPCIONES SOBRE EL PROCESO PARA ACCEDER A LOS SERVICIOS DE SALUD}

En esta sección se presentan los hallazgos del proceso de recolección de información a través de los grupos focales realizados con personas migrantes y con organizaciones que brindan asistencia a esta población, los procesos de mapeo participativa y cartografía corporal y el análisis documental de la directriz y circulares que protegen su derecho a la salud.

\section{Normativa cambiante, desinformación y una ruta engorrosa}

A partir de la información recabada con la población refugiada y solicitante y con las organizaciones, se encontraron varios hallazgos en relación con los aspectos que se tornan determinantes para navegar por la institucionalidad pública y acceder a los servicios de salud. Primeramente, contar con un documento de identidad oficial se constituye como el primer criterio de acceso y a su vez, como el primer obstáculo al que se enfrentan. De acuerdo con la circular ACE-128-07-2019, sobre el Registro de Protecciones con Cargo al Estado 
en el Sistema de Información y Agendas emitida por la CCSS, la población deberá presentar su pasaporte, carné o documento emitido por la Dirección General de Migración y Extranjería. Sin embargo, en muchos casos este es un requisito con el que la población no puede cumplir.

Los retrasos en los procesos para obtener una cita para solicitar la condición de refugio ante la DGME han ocasionado que una gran cantidad de personas aún no cuenten con el carné de solicitante. Las organizaciones que brindan atención estiman que se reportan retrasos de hasta seis meses. En este sentido, aunque los solicitantes se acercan a los EBAIS con la fecha de la cita agendada, el acceso resulta denegado por no portar un documento que contenga una fotografía y en otros casos, porque lo funcionarios no reconocen el documento de solicitante de refugio (grupos focal 2 con representantes de organizaciones que asisten migrantes, comunicación personal, 7 de octubre de 2019).

Este retraso en el proceso para acceder a la documentación se constituye a su vez en una barrera para acceder a otros servicios y, si bien existen mecanismos para que una persona sin documento de identificación pueda ser atendida, en la práctica, muchas veces resultan poco efectivos. Por ejemplo, si la persona carece de un documento vigente tienen que dirigirse al área de Registros MédicosAdscripción (REDES) dentro de lo que se conoce como Validación de Derechos, en los centros de salud, para que se les asigne un número interno y puedan atenderles. No obstante, como denuncian las organizaciones que atienden a la población, se trata de un área de difícil acceso.

"Hemos tenido muchos casos de negación de acceso en los casos, sobre todo, de personas que no tienen el carnet y lo que pasa con estos casos es que en las directrices se habla de una cuestión que se llaman REDES, eso no existe realmente, no existe como lo decían las circulares, REDES son los de registro y esos de registro son los de ventanilla, o sea no es como una oficina física que es lo que parece que dice la circular, eso no existe y en la Caja no es fácil acceder, como a la inmediatez" (grupos focal 2 con representantes de organizaciones que asisten migrantes, comunicación personal, 7 de octubre de 2019).

Uno de los aspectos más relevantes identificados en los grupos focales, es que tanto las organizaciones que trabajan en la atención 
de la población como las personas solicitantes y refugiadas, perciben que la ruta para acceder a los servicios es sumamente compleja, la describen como "una telaraña institucional" "llena de trabas", "sumamente engorrosa" y sobre la que no hay suficiente información orientadora (grupo focal 1 con población solicitante de refugio, grupo focal 2 con representantes de organización que trabaja con migrantes, grupo focal 3 con población solicitante de refugio , comunicación personal, 2 de octubre de 2019, 7 de octubre de 2019, 11 de diciembre de 2019).

De acuerdo con las personas refugiadas y solicitantes entrevistadas, el proceso para adscribirse a la Caja es largo, burocrático y complejo. La ausencia de protocolos respecto a la atención de la población solicitante de refugio a lo interno de la institución, las diferencias en el manejo de información entre los distintos funcionarios y la poca orientación que reciben la población sobre cómo completar los procesos, a cuáles instancias dirigirse, entre otros aspectos dificultan, su acceso a los servicios. En cuanto al manejo de información diferenciado, muchos funcionarios de ventanilla desconocen las directrices y circulares que regulan su acceso a los servicios, de manera que, aunque en la normativa se establece el derecho, continúan denegándolo:

"Algo que nos pasa siempre es que en el EBAIS les dicen que tienen que tener un seguro, cosa que la directriz de MIDEPLAN establece que no es así, solo se necesita a partir del segundo nivel de atención. En el primer nivel vos podés llegar sin seguro y recibir la atención médica integral, hacerte los chequeos en salud, únicamente presentando el documento" (grupo focal 4 con representantes de organización que trabaja con migrantes, comunicación personal, 3 de febrero de 2020).

Este desconocimiento se agrava porque, como reportan las organizaciones, las normativas cambian de manera constante, no siempre tienen una redacción clara y algunas imponen mayores restricciones y otras facilitan el acceso, de manera que darle seguimiento a la ruta que debe seguir la población para acceder a los servicios se torna complejo.

"Había en un momento una circular que en la que se establecía atención por igual para personas hasta los 25 años, era como parte de la Ley del Adulto Joven entonces había una cobertura de 18 a 25 aunque no estuvieran asegurados y esa la logramos usar con 
varia gente pero ya llegó el momento en el que dejó de funcionar; entonces como que va cambiando así (...) esas directrices salen de repente, hoy sale y es ley u orden de ley y luego cambian" (grupo focal 2 con representantes de organización que trabaja con migrantes, comunicación personal, 7 de octubre de 2019).

De acuerdo con la información recopilada, la población indica que la información sobre la ruta que deben seguir para acceder a los servicios la obtienen de las organizaciones no gubernamentales que les atienden o de las propias redes que construyen entre los grupos de población migrante. Incluso, como se reporta en varios de los grupos focales, son ellos mismos, quienes muchas veces tienen que informar a los funcionarios de los centros de salud, sobre la normativa existente

"A mí me negaron el acceso al servicio, llegó una funcionaria, supuestamente la jefa del EBAIS, a decir que no sabía de la directriz. Lo sabía el área de Validación y ella no lo sabía. Entonces agarro y le saco la directriz porque la andaban impresa y le expliqué que ahí dice que me tiene que dar una autorización para una cita" (grupo focal 2, con representantes de organización que trabaja con migrantes, comunicación personal, 7 de octubre de 2019).

Casos similares al anteriormente expuesto fueron recurrentemente mencionados en los grupos focales. El perfil de las personas migrantes que llegaron al país a partir de este nuevo flujo migratorio tiene una significativa formación política (de hecho, la gran mayoría son activistas a favor de los derechos humanos, con una importante sensibilidad de género, de respeto y defensa de la diversidad, inclusión social y solidaridad) y en algunos casos un nivel educativo alto, que les permite responder a través distintos recursos ante las barreras que enfrentan para acceder a los servicios. De esta forma, los nuevos grupos migratorios que llegaron al país hacen uso de su capital humano para intentar superar algunos de los obstáculos en el acceso al introducir demandas al sistema de salud y específicamente direccionarlas hacia los operadores, con el fin de que se protejan y garanticen los derechos que tienen.

\section{Temor por el cobro de los servicios}

Uno de los aspectos que se evidenció de forma recurrente en los grupos realizados, es que existe un temor por parte de la población al cobro de servicios, que se basa en la falta de claridad sobre 
lo dispuesto en las distintas circulares mencionadas y la forma en que opera el cobro al Estado o a las diferentes instancias y fondos gubernamentales. Como se mencionó de forma previa, a partir de las directrices, ACE-157-08-18 y ACE-128-07-2019, se señala que las personas solicitantes podrán inscribirse en el servicio REDES, utilizar los servicios y posteriormente, esos servicios pueden ser facturados a nombre de la persona, pero finalmente cubiertos por las instancias gubernamentales señaladas en las directrices. La disyuntiva, tanto para la población como para las organizaciones continúa siendo en cuáles casos se aplicará un cobro directo y en cuáles no, ya que las directrices no son claras y algunos funcionarios emiten la factura sin que luego sea cubierta por el Estado.

Según se reportó en los grupos focales, la decisión final sobre la emisión del cobro recae en los operadores de las instituciones y puede estar influenciada por aspectos tan diversos como la cantidad de población migrante que recibió el centro de salud durante el día, los prejuicios que tienen los funcionarios sobre los nicaragüenses y su disposición o no en aplicar la normativa vigente. Como reportan las personas refugiadas y solicitantes entrevistadas, en muchos casos los cobros se emiten y debido a que no tienen capacidad de pago para cubrirlos, deben recurrir a las organizaciones no gubernamentales con el fin de apelarlos (grupo focal 4 con representantes de organización que trabaja con migrantes, comunicación personal, 3 de febrero de 2020).

Como señalan las organizaciones entrevistadas, la falta de información estandarizada sobre cómo funciona el proceso de cobros, inhibe a algunas personas migrantes de tratarse alguna dolencia o malestar con el fin de evitar una deuda que, como reconocen, difícilmente podrán pagar.

"Muchos funcionarios dicen: bueno le podemos dar la cita, pero se le va a facturar a su nombre y entonces pasa que la gente se va porque no saben ni el monto, pero realidad no es a la persona que se le va a cobrar, sino que el funcionario necesita emitir un número utilizando los datos persona, pero al final se le va a cobrar al Estado" (grupo focal 2, con representantes de organización que trabaja con migrantes, comunicación personal, 7 de octubre de 2019).

Lo anterior se manifestó de forma reiterada en los grupos focales. Se trata de una cultura de cobro interiorizada en el personal de ventanillas que pretende el pago de toda atención, debilitando 
la vocación de atención universal que está en la misión de la seguridad social costarricense. La falta de conocimiento que tiene un sector de la población migrante sobre cómo funciona la cobertura del seguro social, también han generado que personas adquieran deudas sustantivas al desconocer que algunas poblaciones no requieren contar con un aseguramiento para acceder a los servicios, como los menores de edad y al no haber sido debidamente orientadas, terminan con deudas dentro de la Caja que no pueden pagar y que les impiden asegurarse posteriormente (grupo focal 4 con representantes de organización que trabaja con migrantes, comunicación personal 3 de febrero, 2020).

“Tenemos el caso de un señor que se adscribió a la modalidad de trabajador independiente porque su a hija menor de edad le detectaron leucemia. Entonces él en su desconocimiento optó por una modalidad de seguro sin saber que a su niña la cubría el seguro por el Estado. Él cae en mora por un año aproximadamente, esta mora lo que genera es que a la niña las atenciones médicas se las fueran cobrando al asegurado, es decir a su papá, entonces el señor tiene una deuda de diez millones que pudo haber asumido el Estado" (grupo focal 4 con representantes de organización que trabaja con migrantes, comunicación personal, 3 de febrero de 2020).

El temor por el cobro a los servicios no solo se identifica en las personas migrantes, sino también en los funcionarios de los centros de salud, precisamente por la falta de conocimiento sobre las directrices. Según se reportó en uno de los grupos focales, hay funcionarios que le han expresado a la población que no pueden brindarles la atención porque les preocupa que sean ellos mismos quienes tengan que asumir los gastos por la atención brindada (grupo focal con población solicitante de refugio, comunicación personal, 2 de octubre de 2019).

\section{Xenofobia institucionalizada}

En los grupos focales, de cartografía corporal y mapeo participativo que se realizaron, la población mencionó reiteradamente que la CCSS es una de las instituciones donde más han percibido discriminación y han recibido maltrato. Según señalan se trata de una "xenofobia institucionalizada", permeada en todos los niveles de la institución. De acuerdo con las personas entrevistadas en uno de los grupos con poblaciones solicitantes de refugio, los funcionarios

\section{1}


les han negado la atención porque sienten aporofobia ${ }^{12}$ hacia los nicaragüenses, lo que se expresa en la forma despectiva con la que los atienden, el trato irrespetuoso al que recurren cuando se les hacen consultas y en las reiteradas ocasiones en las que les deniegan el acceso a los servicios (grupo focal 1 con población solicitante de refugio, grupo focal 3 con población solicitante de refugio, comunicación personal, 2 de octubre de 2019 y 11 de diciembre de 2019). Según sus testimonios, los funcionarios de ventanilla tienen prejuicios contra la población nicaragüense y estos se manifiestan con mayor intensidad cuando las personas tienen menores recursos económicos hacen consultas y en las reiteradas ocasiones en las que les deniegan el acceso a los servicios (grupo focal 1 con población solicitante de refugio, grupo focal 3 con población solicitante de refugio, comunicación personal, 2 de octubre de 2019 y 11 de diciembre de 2019).

En las experiencias compartidas en los grupos focales se enfatizó que tanto el personal administrativo como el personal de salud de la CCSS muestra actitudes de rechazo hacia la población nicaragüense en el ejercicio de sus funciones y que estos tratos se detienen, únicamente, cuando la población amenaza con repercusiones legales en su contra, como imponerles una demanda (grupo focal 3 con población solicitante de refugio, comunicación personal, 11 de diciembre de 2019). De esta forma, la población percibe que la herramienta con la que cuentan para hacer frente a este fenómeno de "xenofobia institucionalizada" es recurrir a sus conocimientos en materia de derechos, adquiridos a partir de su trayectoria activista, con el fin de interponer las denuncias ante las instancias correspondientes. A estos efectos, el papel de las organizaciones que brindan atención a la población ha sido fundamental, ya que les orientan sobre cómo acudir a las Contralorías de Servicios (grupo focales 1 con población solicitante de refugio, grupo focal 3 con población solicitante de refugio, comunicación personal, 2 de octubre de 2019 y 11 de diciembre de 2019).

\section{Un proyecto universalista con limitaciones}

En los grupos focales, la población refugiada y solicitante expresó que, al llegar a Costa Rica, se enfrentaron con una realidad ines-

12 Término acuñado por la filósofa Adela Cortina para definir el rechazo a la persona en situación de pobreza (Cortina,2017). 
peradamente adversa, que tuvo como una de sus más grandes manifestaciones los problemas que han enfrentado para acceder a servicios de salud en la CCSS. Según señalan, han tenido una experiencia muy distinta a la expectativa idealizada que traían de Costa Rica como una sociedad hospitalaria e inclusiva para quienes necesitaban refugio. Esta población, ha encontrado en la institución una gran cantidad de barreras para acceder a los servicios de salud $\mathrm{y}$ reportan que han sido las organizaciones no gubernamentales, y no el Estado costarricense, quienes se han encargado de brindarles la orientación necesaria para superarlas o al menos para encontrar nuevas rutas de acceso que les permitan atender sus necesidades en salud de forma oportuna.

Según señalan, a partir de su experiencia en Costa Rica han identificado no solo que la CCSS enfrenta importantes restricciones financieras que pueden tener efectos sobre sus posibilidades de acceso, sino que, además, el derecho a la salud está comprometido para la población nacional también. Como expresó uno de los participantes de los grupos focales "(s)i estas personas aseguradas y de nacionalidad costarricense los atienden de manera deficiente o no los atienden, entonces de seguro a mí por mi situación de solicitante o de refugiado no me atenderán" (grupo focal 3 con población solicitante de refugio, comunicación personal, 11 de diciembre de 2019).

Lo anterior enfatiza en la relevancia de desarrollar mayor investigación sobre posibles manifestaciones de erosión del universalismo en el país y las afectaciones que esto genera sobre el acceso a la salud en la población migrante, ya que, ante escenarios de mayor presión financiera, mayores demandas insatisfechas, reducción de la calidad y equidad en el acceso a los servicios, las políticas restrictivas y las prácticas excluyentes dentro de la seguridad social contra la población migrante, podrían incrementarse. En este sentido, analizar lo que ocurre con la población migrante en materia de salud, no solo requiere incorporar el estudio sobre sus percepciones en cuanto al acceso a los servicios de salud, sino también colocar sus experiencias en una discusión más amplia que tome en consideración cómo la política social vigente y las transformaciones de la seguridad social, tienen implicaciones sobre el ejercicio efectivo de sus derechos. 


\section{CONCLUSIONES}

A partir de la información recopilada en este artículo, se pone de manifiesto que, a pesar la tradición solidaria y de aspiración a una cobertura universal que en materia de salud ha tenido el país, existe una gran cantidad de barreras institucionales que impiden a la población migrante acceder a los servicios. Las nuevas directrices y circulares para atender a la población nicaragüense que ingresó a partir de abril de 2018 abren rutas de acceso a los servicios de salud que en la práctica se ven obstaculizadas por desinformación de los funcionarios, cambios frecuentes en la normativa, poca precisión en su redacción y trámites engorrosos para completar los procesos de acceso y niveles importantes de xenofobia institucionalizada.

Los testimonios de la población refugiada y solicitante evidencian una brecha importante entre las directrices emanadas desde la Junta Directiva y otras autoridades de la CCSS hacia los niveles operativos que atienden de manera directa a la población solicitante de refugio. Además, se evidencia la existencia de una cultura de cobro interiorizada en el personal de ventanillas que pretende el pago de toda atención, debilitando en la práctica la vocación de atención universal que está en la misión de la seguridad social costarricense y en este tema no solo para la población migrante, pero en este caso la información analizada brinda indicios de que la actitud de rechazo es más fuerte hacia la población extranjera. Tercero, hay una actitud xenofóbica en parte del personal de seguridad social, a veces abierta y a veces encubierta, que ha sido identificada por la población entrevistada.

Ante estas barreras, se identificó que las organizaciones no gubernamentales que dan apoyo a la población migrante han desempeñado un papel protagónico en la búsqueda de alternativas para resolver los nudos que impiden el acceso a los servicios y para defender los derechos de esta población cuando son violentados por la institucionalidad pública. Como parte de sus estrategias, han recurrido a los acompañamientos individuales y a la difusión de información sobre las directrices, así como a estrategias de incidencia para que se generen cambios en la respuesta estatal. Sin embargo, estas organizaciones reconocen que se requiere impulsar procesos de sensibilización con mayor alcance a nivel nacional, debido a la a la gran cantidad de centros de salud.

Las personas autoras considera necesario en el futuro hacer un 
esfuerzo para lograr una concordancia efectiva entre la misión institucional de lograr una cobertura universal del acceso al servicio de salud y la lógica operativa en la que prevalecen prácticas de exclusión y rechazo a la atención sobre la base del cobro del servicio. Además, también se considera importante impulsar un proceso de información sobre las directrices de atención a la población refugiada entre todo el personal de salud, al igual que su sensibilización sobre la importancia de garantizar la salud independientemente de su nacionalidad.

\section{FUENTES CONSULTADAS}

Alto Comisionado de Naciones Unidas para los Refugiados. (2020). Convenio ACNUR - CCSS. Recuperado de: https://help.unhcr.org/costarica/convenio-acnur-ccss

Alvarenga, X., Fernández, A., Fernández, D. y Peña, D. (2018). Política social inclusiva y servicios privados de salud en Costa Rica: encuentros, desencuentros y retos. [Tesis de Licenciatura, Universidad de Costa Rica]. Recuperado de: http://www.kerwa.ucr.ac.cr/handle/10669/74768.

Añón, M.J. (2002). Ciudadanía social: La lucha por los derechos sociales. Cuadernos Electrónicos de Filosofía del Derecho. 6. Recuperado de: https://www.uv.es/ afd/CEFD/6/anyon.htm

Bravo, J. (16 de setiembre de 2017). Costa Rica con pocas trabajadoras domésticas aseguradas. La Prensa. https://www.laprensa.com.ni/2017/09/16/ economia /2297898-costa-rica-con-pocas-trabajadoras-domesticas-aseguradas\#: :text $=$ Nicaragua $\% 20 \mathrm{el} \% 20$ principal $\% 20$ proveedor\%20de $\% 20$ trabajadoras $\% 20$ dom $\%$ C3\%A9sticas\&text $=$ La $\% 20$ Enaho\%20encontr\%C3\%B3\%20durante $\% 20$ el,emplea $\% 20$ en $\% 20$ el\%20servicio\%20dom\%C3\%A9stico.

Carrillo, R., Martínez Franzoni, J., Naranjo, F. y Sauma, P. (2011). Informe del equipo de especialistas nacionales nombrado para el análisis de la situación del seguro de salud de la CCSS. Recomendaciones para restablecer la sostenibilidad financiera del seguro de salud. San José: CCSS.

Chamorro Barrios, C.F. (2020). Nicaragua: antes y después de la Rebelión de Abril. En: Cortés Ramos, A., López Baltodano, U. y Moncada Bellorín, L. (eds.). Anhelos de un nuevo horizonte. Aportes para una Nicaragua democrática. San José: FLACSO, pp. 23-42. Recuperado de: https://bit. ly/ANH2020.

\section{5}


Cordero Parra, M. (30 julio de 2019). Tiempos de espera en la CCSS mantienen en largo suplicio a los asegurados. Semanario Universidad. https:// semanariouniversidad.com/pais/tiempos-de-espera-en-la-ccss-mantienen-en-largo-suplicio-a-los-asegurados/

Cortés Ramos, A. (2020). Informe Final. Programa de capacitación y educación continua de la Escuela de Ciencias Políticasm ED3311. San José: Escuela de Ciencias Políticas. Recuperado de: https://www.dropbox. com/s/3p78nrl755dwbc9/Informe\%20Final.pdf?dl=0

Cortés Ramos, A. (8 de agosto de 2009). Producción histórica de las dinámicas migratorias entre Nicaragua y Costa Rica. Ponencia de Conferencia Magistral en el Centro de Investigación en Identidad y Cultura Latinoamericana de la Universidad de Costa Rica.

Cortés Ramos, A., López Baltodano, U. y Moncada Bellorín, L. (eds.). (2020). Anhelos de un nuevo horizonte. Aportes para una Nicaragua democrática. San José: FLACSO. Recuperado de: https://bit.ly/ANH2020.

Contraloría General de la República. (2018). Encuesta Nacional de Servicios Públicos. Contraloría General de la República. Recuperado de: https:// cgrfiles.cgr.go.cr/publico/docsweb/enpsp-2018/resultados.html

Cortina, A. (2017). Aporofobia: el rechazo a un pobre. Un desafío para la democracia. Madrid: Paidós Estado y Sociedad.

Dirección General de Migración y Extranjería. (2020). Informes de Unidad de Refugio mayo 2018-abril 2020. San José: Unidad de Refugio, Dirección General de Migración y Extranjería.

ENAHO. (2017). 2010-2017. Población total por condición migrante según año y edad. Tomado de: https://www.inec.cr/documento/enaho-20102017-poblacion-total-por-condicion-migrante-segun-ano-y-edad

Hidalgo, K. (1 de agosto de 2020). Decreto pretende hacer públicos los datos de las listas de espera de la CCSS. Autoridades rindieron cuentas este jueves ante la Sala IV. Amelia Rueda. https://www.ameliarueda.com/nota/ ccss-listas-espera-decreto-datos-sala-magistrada-pacientes-asegurados

Hirschman, A. (1969). Exit, Voice and Loyalty. Massachusetts: Harvard University Press.

La Nación. (1 de abril de 2019). Sala IV da seis meses a la CCSS para elaborar un plan que reduzca listas de espera. La Nación: https://www. nacion.com/el-pais/salud/sala-iv-da-meses-a-la-ccss-para-elaborarplan-que/KSSWAUWAXNHSBDZBR5H7UBRQBY/story/

Marshall, T. H. and Bottomore, T. (1992). Citizenship and Social Class Cam- 
bridge: Cambridge University Press.

Martínez Franzoni, J. y Sánchez-Ancochea, D. (2012). Good jobs and social services: How Costa Rica achieved the elusive double incorporation. . Hampshire: Palgrave Macmillan.

Martínez Franzoni, J. y Sánchez-Ancochea, D. (2016) The Quest for Universal Social Policy in the South: Actors, Ideas and Architectures. Cambridge: Cambridge University Press.

Miranda, G. (1994). La Seguridad Social y el Desarrollo en Costa Rica: San José: Editorial Nacional de Salud y Seguridad Social EDNASS.

Miranda Aburto, W. y Regidor, C. (10 de febrero de 2020) Los nicas en Costa Rica: migrantes históricos. Confidencial. https://confidencial. com.ni/los-nicas-en-costa-rica-migrantes-historicos/

Mohs, E. (1983). La salud en Costa Rica. San José: Editorial Universidad Estatal a Distancia. Recuperado de: https: / / repositorio.binasss.sa.cr/repositorio/bitstream/handle/20.500.11764/12/doc8.pdf?sequence $=1$

Mora, S. (2015). Las luchas por la defensa del derecho a la salud durante el año 2012. Entre concepciones y agendas políticas de las organizaciones laborales de la Caja Costarricense de Seguro Social (CCSS) (Tesis de maestría). Universidad Nacional (UNA), Costa Rica

Morales, A., Acuña, G., y Li Wing-Ching, K. (2010). Migración y salud en zonas fronterizas: Nicaragua y Costa Rica. Santiago: Comisión Económica para América Latina (CEPA) y Fondo de Población de las Naciones Unidas (UNFPA). Recuperado de: https://core.ac.uk/display/38673183

Nuevo Diario. (29 de setiembre de 2017) Costa Rica necesita 100.000 trabajadores agrícolas. Revista Summa. https://revistasumma.com/costa-rica-necesita-100-000-trabajadores-agricolas/

OCDE. (2017). Estudios de la OCDE sobre los Sistemas de Salud. OCDE. Recuperado de: https://www.oecd.org/els/health-systems/Estudios-OCDE-sobre-los-Sistemas-de-Salud_Costa-Rica_Evaluaci\%C3\%B3n-y-Recomendaciones.pdf

OCDE. (2018). Cómo los inmigrantes contribuyen a la economía de Costa Rica. Paris: OCDE Publishing. Recuperado de: https://doi.org/10.1787/9789264303867-es.

Organización Panamericana de la Salud (OPS). (2011). Informe sobre el Estado de Situación Financiera del Seguro de Salud de la Caja Costa- 
rricense del Seguro Social. Situación Reciente y Proyecciones. Washington: OPS

Picado, G. y Salazar, V. (2004). Análisis de la cobertura del seguro de salud a partir del censo de población 2000. San José: Instituto Nacional de Estadística y Censos. Recuperado de: http://www.inec.go.cr/sites/default/files/documetos-biblioteca-virtual/ansimcenso2000-15.pdf

Recio, P. (1 de abril de 2019). Sala IV da seis meses a la CCSS para elaborar un plan que reduzca listas de espera. La Nación. https:/ /www.nacion.com/ el-pais/salud/sala-iv-da-meses-a-la-ccss-para-elaborar-plan-que/ KSSWAUWAXNHSBDZBR5H7UBRQBY/story/

Resolución № 05560 - 2019. 2019. 18-014499-0007-CO (Sala Constitucional, 29 de marzo).

Rivas, E. y Regidor, C. (11 de febrero de 2020). Los refugiados de la represión de abril. Confidencial. https://confidencial.com.ni/los-refugiados-de-la-represion-de-abril/

Rosemberg, M. (1979). Las luchas por el Seguro Social en Costa Rica. San José: Editorial Costa Rica.

Sandoval, C. (ed.). (2007). El mito roto. Inmigración y emigración en Costa Rica. San José: Editorial Universidad de Costa Rica.

Sala Constitucional. (marzo, 2019). Resolución № 05560 - 2019. 2019. 18014499-0007-CO . San José: Sala Constitucional de la Corte Suprema de Justicia.

Solís Ramírez, M.I. (11 de julio de 2013). CCSS tiene en funcionamiento 1 014 Ebáis en todo el territorio nacional. Caja Costarricense de Seguro Social. https: / $/$ www.ccss.sa.cr/noticias_antiguas?pagina $=2 \& c a t=22$

Valverde, R. (19 julio 2018). Aumento de migrantes nicaragüenses obliga al Gobierno a buscar ayuda internacional. Semanario Universidad. https:// semanariouniversidad.com/ultima-hora/aumento-de-migrantes-nicaraguenses-obliga-al-gobierno-a-buscar-ayuda-internacional/

Voorend, K. (2019). ¿Un imán de bienestar en el Sur? Migración y política social en Costa Rica. San José: Editorial Universidad de Costa Rica.

AGRADECIMIENTOS. Agradecemos a la población solicitante de refugio que a través de los intercambios desarrollados en el marco del proyecto "Acompañar para integrar", nos han permitido acercarnos a su realidad y nos han orientado para comprender dimensiones sobre su proceso de asentamiento de las que poco se habla, pero cuyo abordaje es fundamental para promover su integración 
local. Lo que nos han compartido y enseñado es invaluable.

También agradecemos el apoyo constante que nos facilitaron las organizaciones no gubernamentales Cenderos, Voces sin Fronteras y RET Internacional con quienes hemos trabajo de manera conjunta durante el último año y cuyos aportes han sido vitales para el desarrollo de esta investigación. Se agradece a la Iniciativa Universitaria por Nicaragua (IUN) por el apoyo y acompañamiento que le han brindado a la población migrante y refugiada nicaragüense, así como por apoyar la realización del curso sobre Ciudadanía, Derechos Humanos y Migración en Costa Rica. Sin su apoyo, este curso no habría sido posible.

Finalmente, expresamos nuestro agradecimiento a la Universidad de Costa Rica, casa de estudios desde la que se articula el proyecto "Acompañar para integrar" y a la Comisión de Acción Social de la Escuela de Ciencias Políticas, cuyo soporte ha sido fundamental para la ejecución de esta iniciativa. Concluimos manifestando nuestra admiración a la población refugiada nicaragüense por su lucha y nuestro agradecimiento por enseñarnos tanto.

ALBERTO CORTÉS RAMOS. Doctor en Geografía por la Universidad de Loughborough, Inglaterra. Catedrático en las Escuelas de Ciencias Políticas y Geografía de la Universidad de Costa Rica e investigador del Centro de Investigación y Estudios Políticos (CIEP).

ADRIANA FERNÁNDEZ CALDERÓN. Licencias en Ciencias Políticas por la Universidad de Costa Rica. Docente en la Escuela de Ciencias Políticas de la Universidad de Costa Rica e investigadora del Instituto de Investigaciones Sociales (IIS). 